\title{
Oleuropein inhibits the proliferation and invasion of glioma cells via suppression of the AKT signaling pathway
}

\author{
MING LIU ${ }^{1}$, JIAN WANG ${ }^{1,2}$, BIN HUANG $^{1}$, ANJING CHEN $^{1}$ and XINGANG LI ${ }^{1}$ \\ ${ }^{1}$ Department of Neurosurgery, Qilu Hospital of Shandong University and Brain Science Research Institute, \\ Shandong University, Jinan, Shandong 250012, P.R. China; ${ }^{2}$ Department of Biomedicine, \\ University of Bergen, 5009 Bergen, Norway
}

Received April 3, 2016; Accepted May 12, 2016

DOI: $10.3892 /$ or.2016.4978

\begin{abstract}
Oleuropein, the main phenolic compound of secoiridoids, has been proven to have inhibitory effects on various types of cancers. However, the antitumor effects and related mechanisms in glioma remain unclear. In the present study, U251 and A172 cells were used to assess the effects of oleuropein. Using cell viability assay, we found that oleuropein greatly inhibited the viability of the U251 and A172 cells. Additionally, flow cytometric apoptosis assay indicated that oleuropein induced the apoptosis of the two cell lines. Consistently, the inhibitory effects of oleuropein on migration and invasion were also observed in vitro. In regards to the mechanism, we found that oleuropein significantly decreased phosphorylation of AKT (p-AKT), accompanied by upregulation of Bax and downregulation of $\mathrm{Bcl}-2$. We also found that there was a decrease in the expression levels of matrix metalloproteinase-2 (MMP-2) and MMP-9 after treatment with oleuropein. Furthermore, a specific phosphatidylinositol 3 kinase (PI3K) inhibitor, LY294002, enhanced the pro-apoptotic and anti-invasive effects induced by oleuropein, which suggested that oleuropein suppressed the growth and invasion of glioma cells via inhibition of AKT activity. Taken together, our results indicated that treatment with oleuropein may be an effective therapy for malignant glioma through suppression of tumor proliferation and invasion by inhibition of the AKT signaling pathway.
\end{abstract}

\section{Introduction}

Malignant glioma, with high mortality and morbidity, accounts for $\sim 10 \%$ of tumors in the central nervous system (1).

Correspondence to: Professor Xingang Li, Department of Neurosurgery, Qilu Hospital of Shandong University and Brain Science Research Institute, Shandong University, 107 Wenhua Xi Road, Jinan, Shandong 250012, P.R. China

E-mail:lixg@sdu.edu.cn

Abbreviations: MMPs, matrix metalloproteinases; PI3K, phosphatidylinositol 3 kinase; GBM, glioblastoma

Key words: oleuropein, antitumor, glioma, AKT
Glioblastoma (GBM) is the most aggressive subtype, characterized by high proliferation, infiltration and invasion (2). Even combined with radiation and chemotherapy after surgical resection, the average lifespan expectancy of patients with GBM is still less than 14 months (3). Therefore, new treatments are needed to improve strategies for controlling glioma.

Oleuropein the main phenolic secoiridoid from the olive tree, has several positive functions for human health. Previous studies have reported that oleuropein possesses antioxidant, anti-inflammatory, antimicrobial, antiviral and neuroprotective activity (4-10). However, it has also revealed some promising antitumor function in various cancer cell lines including melanoma B16 cells, human lymphoblastic cell line Jurkat and human colon adenocarcinoma Caco-2 cells (11-13). Notably, recent studies have indicated that oleuropein inhibits growth and induces apoptosis in human breast cancer MCF-7 cells and prostate cancer cell line LNCaP $(14,15)$. However, there are few studies concerning the pro-apoptotic and antiinvasive effects of oleuropein on glioma cells. In the present study, we aimed to ascertain whether oleuropein exhibits an antitumor effect on human glioma cells.

The Bcl-2 family, including Bcl-2 and Bax, is the central focus in the research of glioma cell apoptosis and Bcl-2 inhibits cell apoptosis while Bax promotes cell apoptosis (16). At the same time, the occurrence of glioma development is closely related with powerful invasion. Numerous studies have found that gliomas mostly spread through the white matter and the extracellular matrix. Damage of the extracellular matrix and cell surface adhesion molecules is the molecular basis of invasion. The matrix metalloproteinase (MMP) family, particularly MMP-2 and MMP-9, can degrade many types of extracellular matrix components, destruct the glioma cell histology barrier and play a key role in the process of invasion and metastasis of glioma cells (17).

In the present study, we investigated the effect of oleuropein on glioma cell apoptosis and invasion and explored which signaling pathway is involved in this process. U251 and A172 cells were utilized to investigate the effects of oleuropein. Our data established that oleuropein suppressed glioma cell proliferation by inducing apoptosis in vitro. We also discovered that oleuropein significantly inhibited the migration and invasion of glioma cells. Furthermore, exploration of the underlying 
mechanisms indicated that oleuropein regulated the expression levels of Bax, Bcl-2 and MMPs by inhibiting AKT activation in the glioma cells. LY294002, a specific AKT inhibitor, enhanced the pro-apoptotic and anti-invasive effects of oleuropein. In conclusion, these novel findings demonstrated that the AKT pathway plays a key role in the antitumor effects of oleuropein in glioma cells by regulating the expression of Bax, Bcl-2, MMP-2 and MMP-9. Thus, oleuropein is a promising chemotherapeutic drug for targeting the AKT pathway.

\section{Materials and methods}

Cell lines, cell culture and reagents. The human malignant glioma cell lines U251 and A172 were obtained from the Chinese Academy of Sciences Cell Bank of Type Culture Collection (CBTCCCAS; Shanghai, China). Cells were cultured in Dulbecco's modified Eagle's medium (DMEM; HyClone, Logan, USA) with $10 \%$ fetal bovine serum (FBS) at $37^{\circ} \mathrm{C}$ in a $5 \% \mathrm{CO}_{2}$ humidified incubator. Oleuropein was purchased from Push Bio-technology, Ltd. (Chengdu, China) and was dissolved according to the manufacturer's instructions. Antibodies against p-AKT, AKT, p-p38, p38, p-ERK, ERK, p-JNK, JNK, GAPDH and HRP-conjugated goat anti-rabbit and anti-mouse IgG were obtained from Cell Signaling Technology (Beverly, MA, USA). Antibodies against MMP-2 and MMP-9 were obtained from ImmunoWay (Newark, DE, USA). Antibodies against p53, caspase-3, caspase-9, Bax, Bcl-2 were obtained from Abcam (Cambridge, UK). LY294002 was purchased from Sigma (St. Louis, MO, USA).

Cell viability assay. Cell viability was detected using the Cell Counting Kit-8 (CCK-8; Guangzhou, China) assay. U251 and A172 $\left(5 \times 10^{3}\right.$ cells/well $)$ cells in $150 \mu 1$ of medium were seeded into 96-well plates. After $12 \mathrm{~h}$ of incubation, the cells were treated with medium containing different concentrations of oleuropein and control cells received culture medium. After a 24-h incubation, the cells were subsequently treated with $20 \mu \mathrm{l}$ of CCK-8 and incubated according to the manufacturer's instructions. The absorbance values were determined at $450 \mathrm{~nm}$ using a microplate reader (Bio-Rad, Hercules, CA, USA).

In vitro scratch assay. The U251 and A172 cells were seeded in 24-well plates. After incubation for $24 \mathrm{~h}$, each well was manually scratched with a $200-\mu 1$ pipette tip, washed lightly with phosphate-buffered saline (PBS) and incubated with oleuropein $\left(0,200\right.$ and $400 \mu \mathrm{M}$, respectively) at $37^{\circ} \mathrm{C}$. After $24 \mathrm{~h}$ of monitoring, the images were captured under a microscope. The distance between two cell edges was statistically analyzed.

Transwell assay. U251 $\left(1 \times 10^{5}\right)$ and A172 $\left(1 \times 10^{5}\right)$ cells were respectively collected in $200 \mu \mathrm{l}$ serum-free medium and were added to the upper chambers. DMEM $(800 \mu \mathrm{l})$ containing $20 \%$ FBS and oleuropein $(0,200$ and $400 \mu \mathrm{M})$ were then added to the lower chamber. After $24 \mathrm{~h}$, the cells that migrated to the lower surface were fixed with methanol and stained with eosin. The number of cells that migrated to the lower side was counted in five random fields under a light microscope. The cell number was counted and statistically analyzed. For the migration assay, the cells $\left(1 \times 10^{4}\right)$ were put into the upper chambers and Matrigel was not used. The other steps were the same as those for the invasion assay.

Apoptosis assay. We used the Annexin V-FITC/PI apoptosis detection kit (BD Biosciences, San Jose, CA, USA) to detect cell apoptosis. Briefly, $1 \times 10^{5}$ untreated or oleuropein-treated cells were collected, respectively. The cells were washed with PBS and put into binding buffer $(500 \mu \mathrm{l})$. Then, the cells were exposed to $5 \mu \mathrm{l}$ Annexin V-FITC and incubated for $15 \mathrm{~min}$ in the dark at $4^{\circ} \mathrm{C}$. Then, $10 \mu \mathrm{l}$ of propidium iodide (PI) was added, and the cells were incubated for $5 \mathrm{~min}$ in the dark. The data were analyzed using WinMDI V2.9 software (The Scripps Research Institute, San Diego, CA, USA).

Western blotting. U251 and A172 cells were treated with 0, 200 and $400 \mu \mathrm{M}$ oleuropein, respectively, for $24 \mathrm{~h}$. Then, the cells were lysed in RIPA buffer containing $1 \%$ protease inhibitors. Equal amounts of proteins from each sample were separated by SDS-PAGE and transferred onto a polyvinylidene fluoride (PVDF) membrane. The membranes were blocked with 5\% non-fat milk for $2 \mathrm{~h}$ at room temperature and incubated with the primary antibodies overnight at $4^{\circ} \mathrm{C}$. Then, the membranes were probed with the appropriate secondary antibodies for $1 \mathrm{~h}$ at room temperature. Finally, the bands were developed using Western Chemiluminescent HRP Substrate and imaged by Image Station 4000MM Pro (Carestream Health Inc., Woodbrige, MA, USA).

Statistical analysis. The results are presented as the mean \pm SD. Statistical differences between groups were analyzed by one-way ANOVA and further Tukey's test using the statistical software of GraphPad Prism 5. All experiments were repeated at least three times. Statistical significance is indicated in the figures as follows: ${ }^{*} \mathrm{P}<0.05,{ }^{* *} \mathrm{P}<0.01$ and ${ }^{* * *} \mathrm{P}<0.001$.

\section{Results}

Oleuropein inhibits the viability of glioma cells. U251 and A172 cells were exposed to different concentrations $(0,100$, 200 and $400 \mu \mathrm{M}$ ) of oleuropein for $24 \mathrm{~h}$, and their viability was analyzed by the CCK- 8 assay. The results showed a stable decrease in cell viability after treatment with oleuropein (Fig. 1A and B). Treatment with $100 \mu \mathrm{M}$ oleuropein had a modest effect, while $400 \mu \mathrm{M}$ oleuropein exhibited an obvious inhibitory effect. The viability of the U251 and A172 cells was reduced to 53.17 and $52.78 \%$ after treatment with oleuropein $(400 \mu \mathrm{M})$ for $24 \mathrm{~h}$, respectively.

Oleuropein suppresses the migration and invasion of glioma cells. We implemented the scratch assay to investigate the effect of oleuropein on the migration of U251 and A172 cells. Migration of the U251 cells was inhibited by oleuropein (Fig. 2A and B). The width of the control group accounted for only $22.72 \%$ of the original width while that of the cells treated with 200 and $400 \mu \mathrm{M}$ accounted for 45.45 and $63.64 \%$ of the original width, respectively. A similar effect was also observed in the A172 cells (Fig. 2C and D). After treatment with 0,200 and $400 \mu \mathrm{M}$ oleuropein, the relative width increased from 38.46 to $73.07 \%$. 

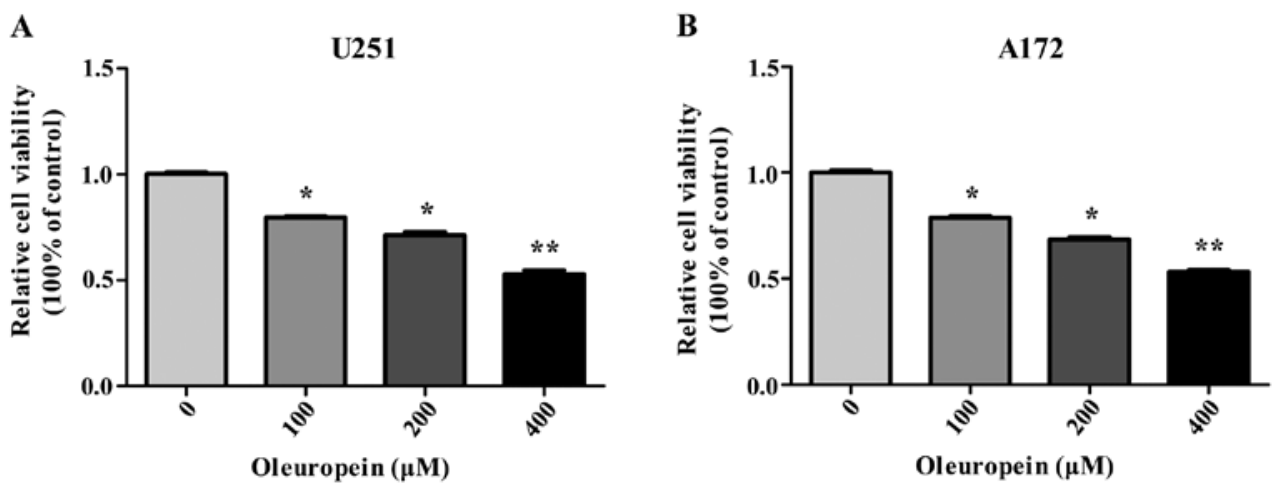

Figure 1. Oleuropein inhibits the viability of U251 and A172 cells in vitro. (A) U251 and (B) A172 cells were treated with different concentrations of oleuropein for $24 \mathrm{~h}$. Oleuropein significantly inhibited the viability of both cell lines in a dose-dependent manner. The results are presented as the means \pm SD of three independent experiments and the corresponding standard error; ${ }^{*} \mathrm{P}<0.05,{ }^{* *} \mathrm{P}<0.01,{ }^{* * *} \mathrm{P}<0.001$ compared with the control group.

A

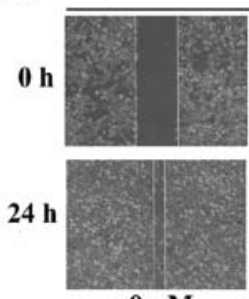

$\mathbf{0} \mu \mathrm{M}$
U251

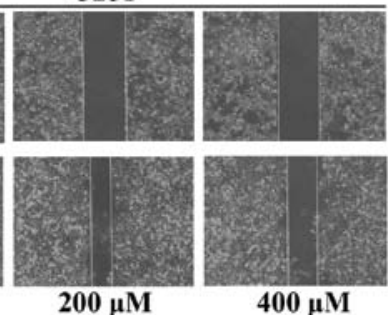

B

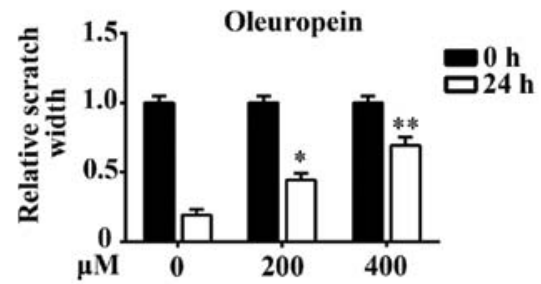

C

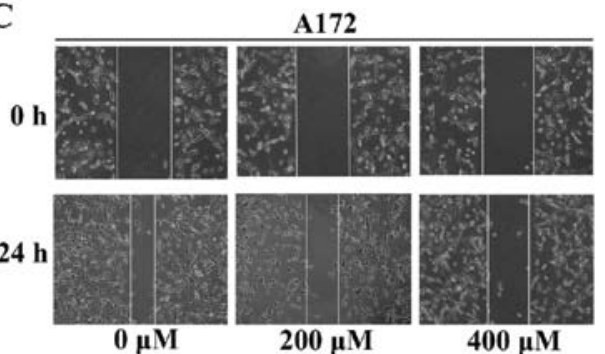

D

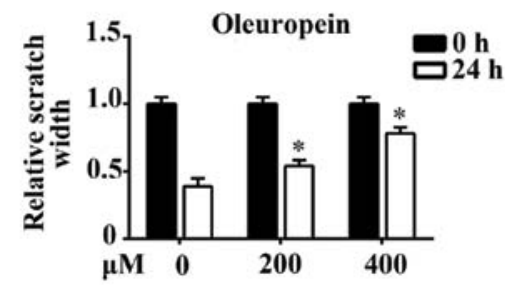

E

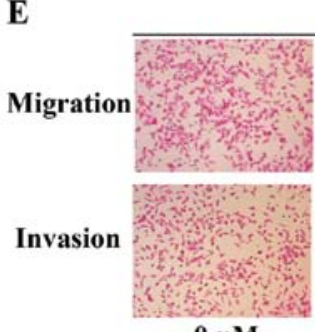

$0 \mu \mathrm{M}$

F

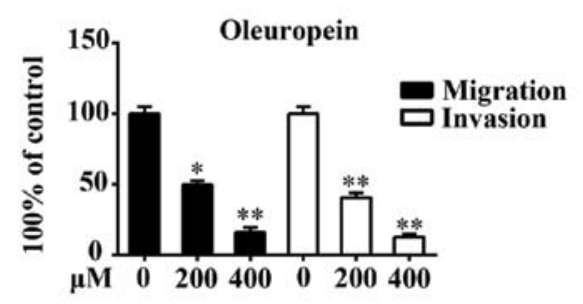

G

Migration

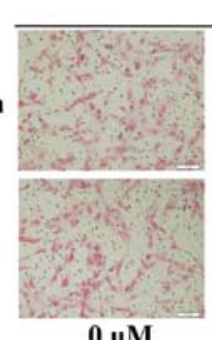

A172

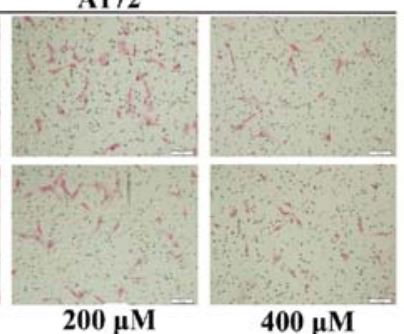

Oleuropein

H

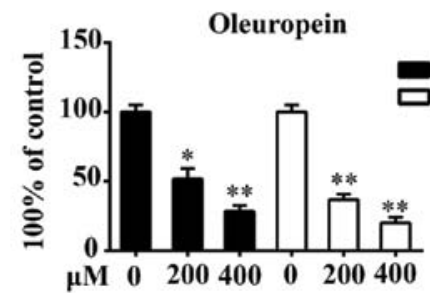

Figure 2. Cell migration and invasion abilities were suppressed by oleuropein. (A) U251 and (C) A172 cells were wounded by scratching with a pipette tip and treated with 0, 200 and $400 \mu \mathrm{M}$ oleuropein for $24 \mathrm{~h}$. The oleuropein-treated (B) U251 and (D) A172 cells showed a lower rate of wound closure than the control cells. (E) The migration and invasion of U251 cells were inhibited after treatment with different concentrations of oleuropein. (F) The number of U251 cells that successfully migrated and invaded was counted. (G) Treatment with oleuropein inhibited the migration and invasion of A172 cells. (H) The decrease in the number of A172 cells indicates the inhibitory effect of oleuropein on cell mobility. Data are presented as the means \pm SD of three independent experiments; "P $<0.05,{ }^{* *} \mathrm{P}<0.01,{ }^{* * *} \mathrm{P}<0.001$.

To test the influence of oleuropein on cell migration and invasion, U251 and A172 cells treated with oleuropein were used to perform the Transwell assay. Our results indicated that oleuropein significantly restrained the migration and invasion 

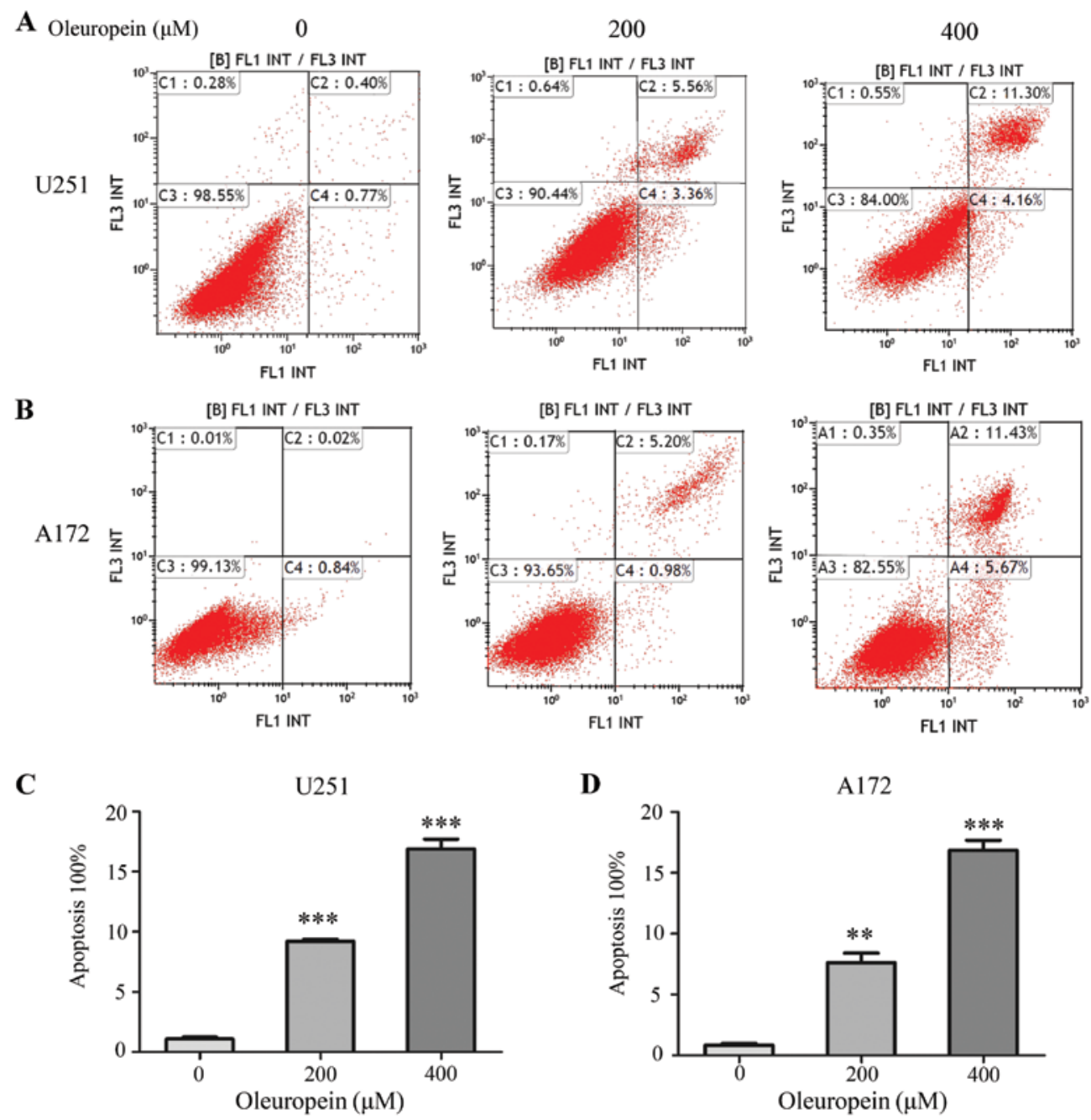

Figure 3. Oleuropein induces dose-dependent apoptosis in U251 and A172 cells. (A) U251 and (B) A172 cells were treated with oleuropein (0, 200 and $400 \mu \mathrm{M}$ ) for $24 \mathrm{~h}$ and stained with FITC-Annexin V and PI. The percentage of surviving cells is shown in the lower left quadrant; and the percentages of early- and late-stage apoptotic cells are shown in the lower right and upper right quadrants, respectively. (C and D) The apoptosis of U251 and A172 cells induced by oleuropein was quantified. Data are presented as the means $\pm \mathrm{SD}$ of three independent experiments; ${ }^{*} \mathrm{P}<0.05,{ }^{* *} \mathrm{P}<0.01,{ }^{* * *} \mathrm{P}<0.001$.

of the U251 cells (Fig. 2E and F), and the number of cells successfully migrating to the lower side in the $400 \mu \mathrm{M}$ group only accounted for $\sim 20 \%$. Furthermore, we observed the same effect on the A172 cells (Fig. 2G and H).

Oleuropein induces apoptosis in U251 and A172 cells. To further detect the apoptotic cells induced by oleuropein, we used Annexin V-conjugated FITC and PI staining to analyze the percentage of apoptotic cells. The lower right (LR) and upper right (UR) quadrants of the histograms show the percentages of early and late apoptotic cells, respectively. The total percentage of apoptotic cells (UR + LR) increased from 1.17 ( $0 \mu \mathrm{M}$ oleuropein) to $8.92 \%$ (200 $\mu \mathrm{M}$ oleuropein), and $15.46 \%$ (400 $\mu \mathrm{M}$ oleuropein) in the $\mathrm{U} 251$ cells, respectively (Fig. 3A and C), while the total percentage of apoptotic cells was increased from $0.86(0 \mu \mathrm{M}$ oleuropein) to $6.18 \%$ (200 $\mu \mathrm{M}$ oleuropein), and $17.10 \%$ (400 $\mu \mathrm{M}$ oleuropein) in the A172 cells (Fig. 3B and D). These results revealed that the treatment of U251 and A172 cells with 200 and $400 \mu \mathrm{M}$ oleuropein for $24 \mathrm{~h}$ promoted apoptosis in a dose-dependent manner.
Effects of oleuropein on the expression of cell apoptosis-related proteins. Numerous studies have reported that pro-apoptotic protein Bax is associated with increased apoptosis, while the anti-apoptotic protein Bcl-2 is associated with the inhibition of apoptosis in target cells $(18,19)$. To investigate the change in Bax and Bcl-2 in the U251 and A172 cells after treatment with oleuropein, western blotting was used to detect the expression of Bax and Bcl-2. The expression of Bax was increased while the expression of Bcl-2 was decreased after treatment with oleuropein (Fig. 4A). Additionally, the expression of cleaved caspase- 9 and -3 were increased (Fig. 4A). Thus, the tumor inhibitory effect of oleuropein on the U251 and A172 cells may be attributed to apoptosis which was mainly regulated by changes in the levels of Bax and Bcl-2.

Effects ofoleuropein on MMP expressionand the AKT pathway. MMPs, particularly MMP-2 and MMP-9, play important roles in breaking down basement membranes and contribute to cancer invasion and metastasis (20). The expression levels of MMP-2 and MMP-9 were significantly decreased after oleuropein treatment (Fig. 4B). However, we found that after 
$\mathbf{A}$

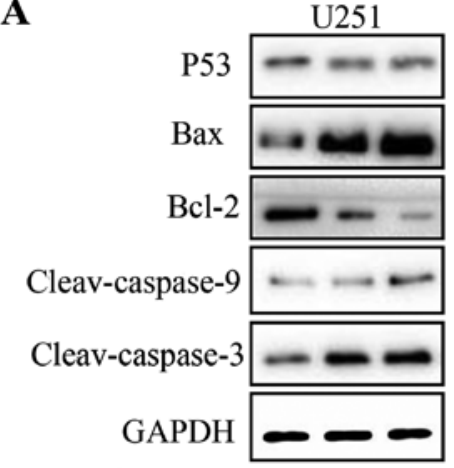

Oleuropein $(\mu \mathrm{M}) \quad 0 \quad 200400$

B

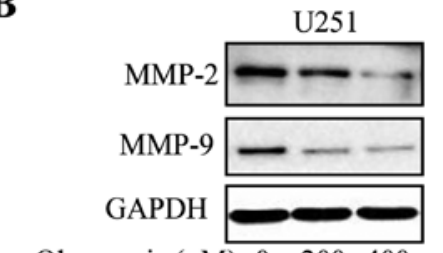

Oleuropein $(\mu \mathrm{M}) \quad 0 \quad 200 \quad 400$

C

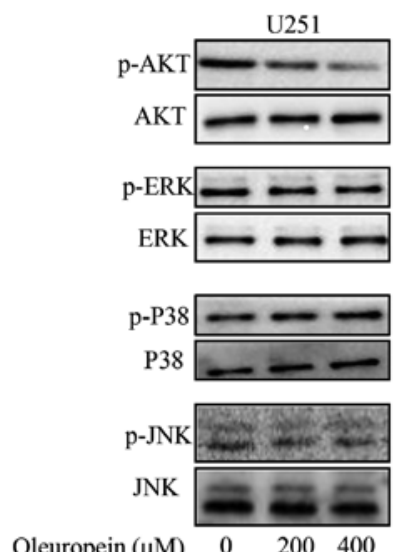

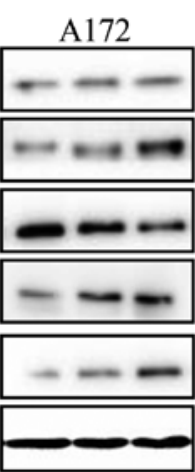

$0 \quad 200400$
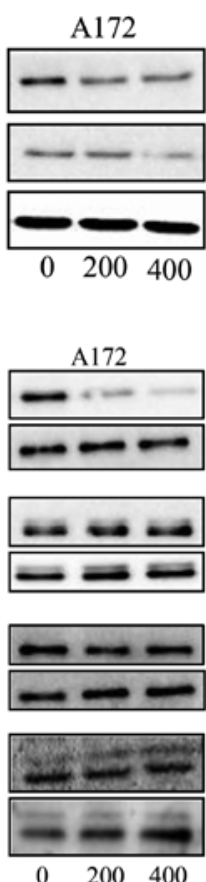

$\begin{array}{lll}0 & 200 & 400\end{array}$

Figure 4. Effects of oleuropein on the levels of apoptosis-related proteins (p53, Bax, Bcl-2, cleaved-caspase-9, cleaved-caspase-3), MMPs (MMP-2 and MMP-9) and several signaling pathway proteins (ERK, JNK, P38 and AKT). (A) The levels of apoptosis-related proteins including P53, Bcl-2, Bax, cleaved-caspase- 9 and cleaved-caspase- 3 were assayed by western blot analysis with GAPDH as a control following treatment with 0,200 and $400 \mu \mathrm{M}$ oleuropein for $24 \mathrm{~h}$ in the $\mathrm{U} 251$ and A172 cells. (B) Changes in the expression of MMP-2 and MMP-9 in the U251 and A172 cells after treatment with 0,200 and $400 \mu \mathrm{M}$ oleuropein for $24 \mathrm{~h}$. (C) MAPK and AKT pathways were examined after 0,200 and $400 \mu \mathrm{M}$ oleuropein treatment for $24 \mathrm{~h}$. The results are representative of at least three independent experiments.

treatment with oleuropein for $24 \mathrm{~h}$, the phosphorylation level of AKT was effectively suppressed in both the U251 and A172 cells, and there were no changes in the expression levels of phosphorylated P38, ERK and JNK (Fig. 4C). Combined with the influence of oleuropein on apoptosis-related proteins, we speculated that AKT signaling may play an important role in the oleuropein-induced pro-apoptotic and anti-invasive effects.

Inhibition of AKT signaling enhances the pro-apoptotic and anti-invasive effects of oleuropein on U251 and A172 cells. LY294002, a pan-PI3K inhibitor, strongly inhibits AKT activation. U251 and A172 cells were cultured in the presence of

either oleuropein $(200 \mu \mathrm{M})$ or/and LY294002 $(50 \mu \mathrm{M})$ for $24 \mathrm{~h}$. The protein levels of p-AKT, AKT, Bax, Bcl-2, MMP-2 and MMP-9 were detected by western blotting. The results revealed that either LY294002 or oleuropein could significantly restrain the expression of AKT, MMP-2, MMP-9 and Bcl-2, and strongly promote the expression of Bax. However, combined treatment with LY294002 played a more significant role in the pro-apoptotic and anti-invasive effects (Fig. 5A and B).

Next, after treatment with oleuropein $(200 \mu \mathrm{M})$ and/or LY294002 $(50 \mu \mathrm{M})$ for $24 \mathrm{~h}$, we used MTT assay to observe the proliferation of the U251 and A172 cells and conducted a Transwell assay to investigate the invasive abilities of the two cell lines. The results indicated that LY294002 cooperated with oleuropein to inhibit proliferation (Fig. 5C and D) and invasion (Fig. 5E-H) of the U251 and A172 cells. In addition, the results revealed that LY294002 can potentiate the pro-apoptotic and anti-invasive effects of oleuropein, which suggests that blocking the AKT signaling pathway may improve the anticancer effects of oleuropein.

\section{Discussion}

Glioma, particularly glioblastoma (GBM) is the leading cause of brain tumor-related death (21). Accumulating evidence has confirmed the anticancer effects of oleuropein against multiple malignant tumors, but the antitumor effects and related mechanisms of oleuropein in glioma are still unclear. In the present study, we demonstrated that oleuropein induced apoptosis and inhibited the invasion of glioma cells by regulating the expression of Bax, Bcl-2, MMP-2 and MMP-9 via suppression of the AKT signaling pathway.

Apoptosis plays vital roles in cell growth with the elimination of aberrant cells (22). Deregulation of apoptosis contributes to pathological conditions and many types of cancers $(23,24)$. The Bcl-2 family consists of several proteins that execute pro- and anti-apoptotic functions and is tightly connected with cancer cell apoptosis, including glioma $(25,26)$. The Bcl-2 family regulates the release of death factors including Smac/ Diablo, apoptosis-inducing factor (AIF) and endonuclease G, which trigger either caspase-dependent or- independent apoptosis (27-30). Bcl-2 is a member of this family and acts as an anti-apoptotic protein, which can indirectly exert effects on cell apoptosis and Bcl-2 locates in the mitochondrial membrane and blocks the internal stores of calcium to be released into the cytoplasm (31-34). In contrast, Bax is a pro-apoptotic protein, which is processed on the outer mitochondrial membrane and it regulates cytochrome $c$ release. Subsequently, activation of caspase-3 causes a series of events related to apoptosis (35-37). Therefore, Bcl-2 and Bax are key factors in apoptosis. In the present study, we examined the changes in Bcl-2 and Bax expression in the U251 and A172 cells following treatment with oleuropein, and found that Bax expression increased and Bcl-2 expression decreased in vitro. However, expression of their downstream substrates, caspase-9 and -3 , were obviously increased. The results indicated that the oleuropein-induced upregulation of Bax expression and downregulation of Bcl-2 expression may cooperate with each other to induce the apoptosis of U251 and A172 cells. We also assessed the expression of P53, which also plays important roles in cell apoptosis, but no significant change was found and we speculated that 
A

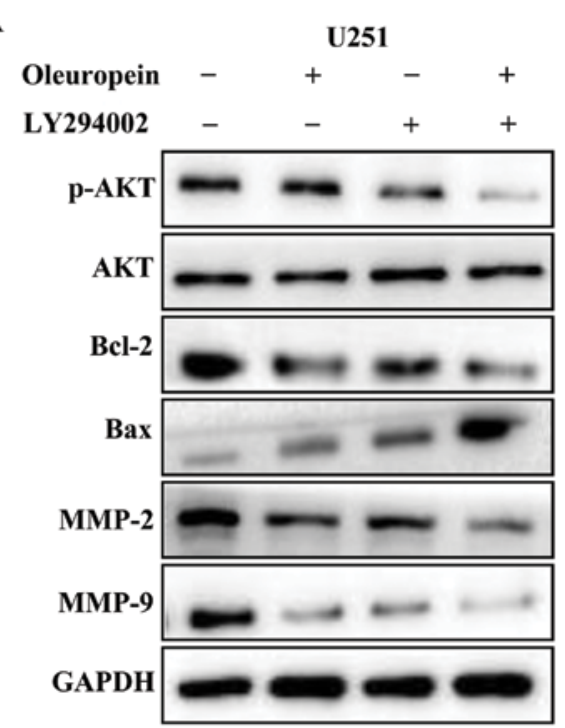

C

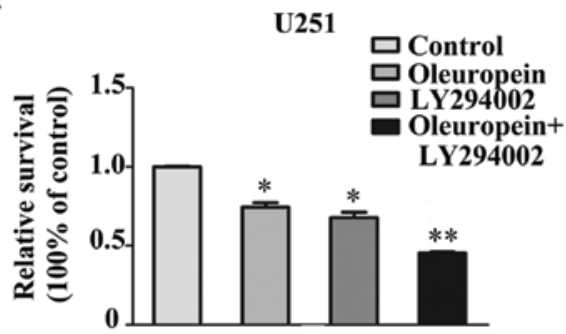

E

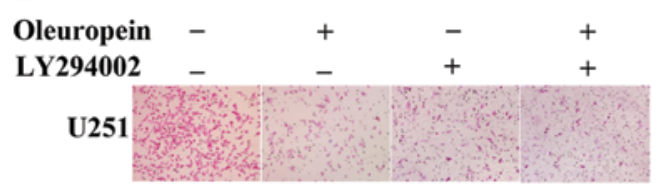

G

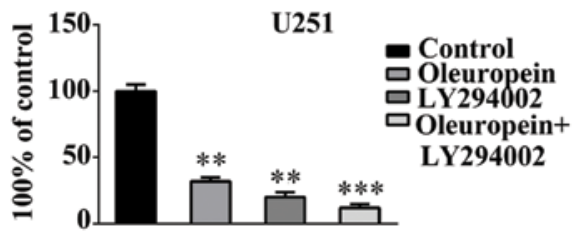

B

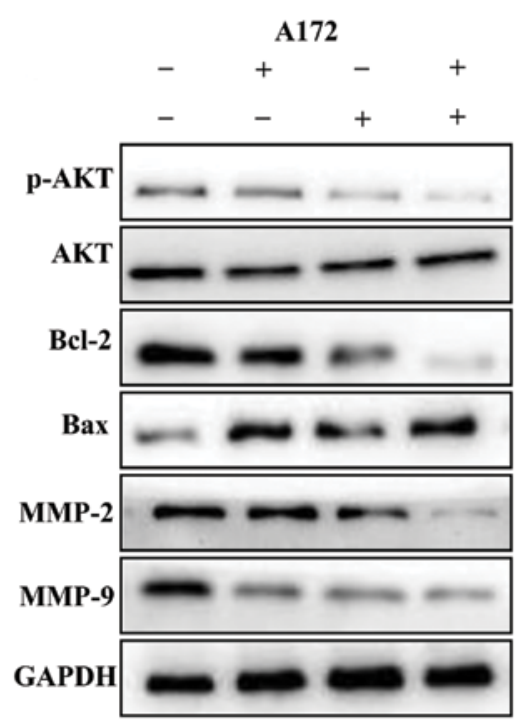

D

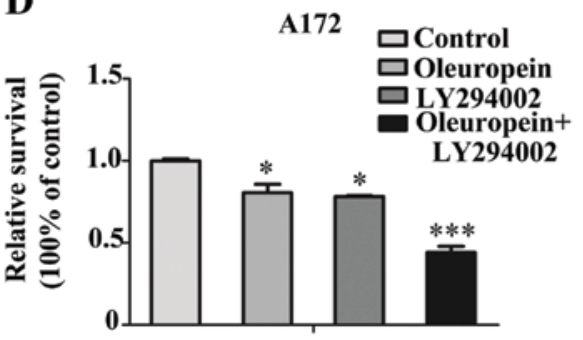

F

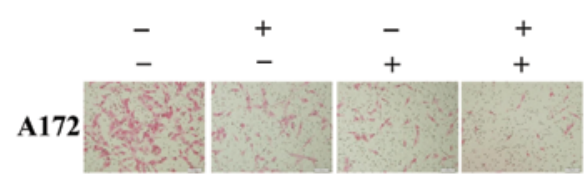

H

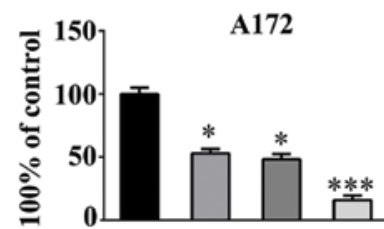

- Control

Oleuropein

나294002

Oleuropein-

LY294002

Figure 5. Inhibition of AKT signaling enhances the pro-apoptotic and anti-invasive effects of oleuropein in glioma cells. (A and B) Expression of p-AKT, AKT, Bax, Bcl-2, MMP-9 and MMP-9 in the U251 and A172 cells after treatment with oleuropein (200 $\mu$ M) and/or LY294002 for 24 h. (C and D) The cell viability was measured by MTT assay. (E and F) U251 and A172 cell invasion was measured by a Transwell assay. (G and H) Transwell assay results of the $\mathrm{U} 251$ and A172 cells were respectively quantified. The results are presented as the means $\pm \mathrm{SD}$ of three independent experiments; ${ }^{*} \mathrm{P}<0.05,{ }^{* * *} \mathrm{P}<0.01,{ }^{* * * *} \mathrm{P}<0.001$, compared with the control cells.

oleuropein-induced apoptosis of U251 and A172 may have little relation with $\mathrm{p} 53$.

The process of invasion and metastasis of glioma is complex and multiple factors may cooperatively be involved. MMPs are considered to be crucial proteinases in tumor invasion and metastasis $(38,39)$. To date, it is widely accepted that degradation of the extracellular matrix and alteration of cell-cell extracellular matrix interactions mainly contribute to the process of invasion and metastasis of tumor cells (20). MMP-2 and MMP-9 proteins are two major members of the MMP family and they are widely involved in cancer metastasis. However, various studies have indicated that patients with increased expression of MMP-2 and MMP-9 proteins have a poor survival (40). MMPs also exert influences on the metastasis and invasion of glioma cells. Various studies have shown that upregulation of MMPs promotes the invasion of glioma stem cells (41). However, downregulation of the expression of MMP-2 and MMP-9 could reduce the invasion of glioma cells (42). Given the effects of MMPs on metastasis and invasion in glioma cells, we tested the changes in MMP-2 and MMP-9 after treatment with oleuropein and found that the expression of MMP-2 and MMP-9 decreased significantly after oleuropein administration in both cell lines in a dosedependent manner. Combined with the Transwell assays, we considered that oleuropein may suppress metastasis and invasion of glioma cells by decreasing the expression of MMP-2 


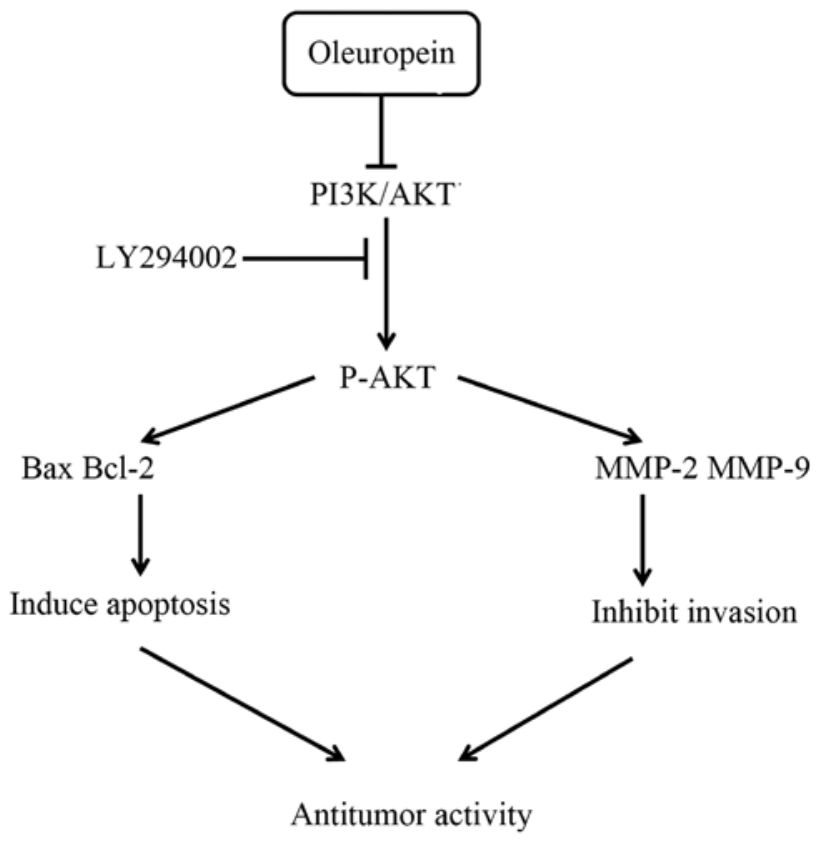

Figure 6. A schematic model: the mechanisms through which oleuropein induces apoptosis and inhibits invasion of glioma cells.

and MMP-9. Given the influence of oleuropein on apoptosisrelated proteins and MMPs, we speculated that some signaling pathway(s) may play an important role in oleuropein-induced inhibition of invasive and promotion of apoptosis.

The synthesis of Bax, Bcl-2, MMP-2 and MMP-9 is regulated by multiple signaling cascades. The AKT signaling pathway participates in many important cellular processes, including tumor cell proliferation, metastasis and invasion (43), and this pathway can also exert influences on the Bcl-2 family and MMPs $(44,45)$. Recent studies indicate that the AKT signaling pathway is overactive in many cancers, and inhibition of this pathway is considered as a novel target for cancer therapy (46). One study demonstrated that oleuropein is implicated as a negative regulator of proliferation of glioma cells (47). However, the underlying mechanisms through which oleuropein exerts its antitumor effects are not clear. In the present study, to further explore the possible mechanism(s) of oleuropein in the inhibition of glioma cell growth, we assessed the levels of phosphorylation of AKT, P38, ERK, JNK in U251 and A172 cells after treatment with oleuropein. We found that after treatment of oleuropein for $24 \mathrm{~h}$, the phosphorylation level of AKT was effectively restrained in both the U251 and A172 cells and there were no significant changes in the activity of phosphorylation of P38, ERK and JNK signaling pathways, which suggested that oleuropein inhibited the activation of AKT. To investigate whether the pro-apoptotic and anti-invasive effects induced by oleuropein are mainly associated with the AKT pathway, LY294002, a PI3K inhibitor, was used. We found that LY294002 alone increased the expression level of Bax and decreased the expression levels of Bcl-2, MMP-2 and MMP-9 while combined use with oleuropein markedly potentiated the pro-apoptotic and anti-invasive effects of oleuropein on the U251 and A172 cells, which suggests that blocking the AKT signaling pathway may improve the anticancer effect of oleuropein on glioma cells. Our data also suggest that AKT may be at the upstream of the pro-apoptotic and anti-invasive process induced by oleuropein (Fig. 6). We speculate that oleuropein may suppress the activation of AKT. Then, expression levels of MMP-2 and MMP-9 are downregulated which inhibits the invasion of glioma. In contrast, the proportion of Bax and Bcl-2 is changed which exerts an effect on the apoptosis of glioma cells.

In conclusion, the present study demonstrated the inhibitory effect of oleuropein on the apoptosis and invasion of glioma cells. The upregulation of Bax and downregulation of Bcl-2 induced by oleuropein were enhanced by suppression of AKT activity. Additionally, the decrease in MMP-2 and MMP-9 induced by oleuropein also attributed to inhibition of the AKT signaling pathway. These findings revealed that the AKT signaling pathway contributes to the anticancer effects of oleuropein on glioma cells and oleuropein may be a new potential therapeutic agent for glioma.

\section{Acknowledgements}

The present study was supported by grants from the Shandong Provincial Outstanding Professional Science and Technology Projects (2015ZDXX0801A01), the Shandong Provincial Outstanding Research and Development Plans (2015GSF118074), and The Fundamental Research Fund of Shandong University (2015QY001).

\section{References}

1. Fisher PG and Buffler PA: Malignant gliomas in 2005: Where to GO from here? JAMA 293: 615-617, 2005.

2. Ricard D, Idbaih A, Ducray F, Lahutte M, Hoang-Xuan K and Delattre JY: Primary brain tumours in adults. Lancet 379: 1984-1996, 2012.

3. Rock K, McArdle O, Forde P, Dunne M, Fitzpatrick D, O'Neill B and Faul C: A clinical review of treatment outcomes in glioblastoma multiforme - the validation in a non-trial population of the results of a randomised Phase III clinical trial: Has a more radical approach improved survival. Br J Radiol 85: 729-733, 2012.

4. Coni E, Di Benedetto R, Di Pasquale M, Masella R, Modesti D, Mattei R and Carlini EA: Protective effect of oleuropein, an olive oil biophenol, on low density lipoprotein oxidizability in rabbits. Lipids 35: 45-54, 2000.

5. Visioli F, Caruso D, Galli C, Viappiani S, Galli G and Sala A: Olive oils rich in natural catecholic phenols decrease isoprostane excretion in humans. Biochem Biophys Res Commun 278: 797-799, 2000.

6. Aziz NH, Farag SE, Mousa LA and Abo-Zaid MA: Comparative antibacterial and antifungal effects of some phenolic compounds. Microbios 93: 43-54, 1998.

7. Bisignano G, Tomaino A, Lo Cascio R, Crisafi G, Uccella N and Saija A: On the in-vitro antimicrobial activity of oleuropein and hydroxytyrosol. J Pharm Pharmacol 51: 971-974, 1999.

8. Micol V, Caturla N, Pérez-Fons L, Más V, Pérez L and Estepa A: The olive leaf extract exhibits antiviral activity against viral haemorrhagic septicaemia rhabdovirus (VHSV). Antiviral Res 66: 129-136, 2005.

9. Lee-Huang S, Zhang L, Huang PL, Chang YT and Huang PL: Anti-HIV activity of olive leaf extract (OLE) and modulation of host cell gene expression by HIV-1 infection and OLE treatment. Biochem Biophys Res Commun 307: 1029-1037, 2003.

10. Bazoti FN, Bergquist J, Markides KE and Tsarbopoulos A: Noncovalent interaction between amyloid-beta-peptide (1-40) and oleuropein studied by electrospray ionization mass spectrometry. J Am Soc Mass Spectrom 17: 568-575, 2006.

11. Mijatovic SA, Timotijevic GS, Miljkovic DM, Radovic JM, Maksimovic-Ivanic DD, Dekanski DP and Stosic-Grujicic SD: Multiple antimelanoma potential of dry olive leaf extract. Int J Cancer 128: 1955-1965, 2011. 
12. Fares R, Bazzi S, Baydoun SE and Abdel-Massih RM: The antioxidant and anti-proliferative activity of the Lebanese Olea europaea extract. Plant Foods Hum Nutr 66: 58-63, 2011.

13. Corona G, Deiana M, Incani A, Vauzour D, Dessì MA and Spencer JP: Inhibition of p38/CREB phosphorylation and COX-2 expression by olive oil polyphenols underlies their anti-proliferative effects. Biochem Biophys Res Commun 362: 606-611, 2007.

14. Han J, Talorete TP, Yamada P and Isoda H: Anti-proliferative and apoptotic effects of oleuropein and hydroxytyrosol on human breast cancer MCF-7 cells. Cytotechnology 59: 45-53, 2009.

15. Acquaviva R, Di Giacomo C, Sorrenti V, Galvano F, Santangelo R, Cardile V, Gangia S, D'Orazio N, Abraham NG and Vanella L: Antiproliferative effect of oleuropein in prostate cell lines. Int J Oncol 41: 31-38, 2012

16. Chan SL and Yu VC: Proteins of the bcl-2 family in apoptosis signalling: From mechanistic insights to therapeutic opportunities. Clin Exp Pharmacol Physiol 31: 119-128, 2004.

17. Nakano A, Tani E, Miyazaki K, Yamamoto Y and Furuyama J: Matrix metalloproteinases and tissue inhibitors of metalloproteinases in human gliomas. J Neurosurg 83: 298-307, 1995.

18. Oltvai ZN, Milliman CL and Korsmeyer SJ: Bcl-2 heterodimerizes in vivo with a conserved homolog, Bax, that accelerates programmed cell death. Cell 74: 609-619, 1993.

19. Marzo I, Brenner C, Zamzami N, Jürgensmeier JM, Susin SA, Vieira HL, Prévost MC, Xie Z, Matsuyama S, Reed JC, et al: Bax and adenine nucleotide translocator cooperate in the mitochondrial control of apoptosis. Science 281: 2027-2031, 1998.

20. Gialeli C, Theocharis AD and Karamanos NK: Roles of matrix metalloproteinases in cancer progression and their pharmacological targeting. FEBS J 278: 16-27, 2011.

21. Kettenmann H, Hanisch UK, Noda M and Verkhratsky A: Physiology of microglia. Physiol Rev 91: 461-553, 2011.

22. Opferman JT and Korsmeyer SJ: Apoptosis in the development and maintenance of the immune system. Nat Immunol 4: 410-415, 2003.

23. Vaux DL, Cory S and Adams JM: Bcl-2 gene promotes haemopoietic cell survival and cooperates with $c$-myc to immortalize pre-B cells. Nature 335: 440-442, 1988.

24. Strasser A, Harris AW, Bath ML and Cory S: Novel primitive lymphoid tumours induced in transgenic mice by cooperation between $m y c$ and $b c l-2$. Nature 348: 331-333, 1990.

25. Yang J, Liu X, Bhalla K, Kim CN, Ibrado AM, Cai J, Peng TI, Jones DP and Wang X: Prevention of apoptosis by Bcl-2: Release of cytochrome $c$ from mitochondria blocked. Science 275: 1129-1132, 1997

26. Danial NN: BCL-2 family proteins: Critical checkpoints of apoptotic cell death. Clin Cancer Res 13: 7254-7263, 2007.

27. Susin SA, Lorenzo HK, Zamzami N, Marzo I, Snow BE, Brothers GM, Mangion J, Jacotot E, Costantini P, Loeffler M, et al Molecular characterization of mitochondrial apoptosis-inducing factor. Nature 397: 441-446, 1999.

28. Du C, Fang M, Li Y, Li L and Wang X: Smac, a mitochondrial protein that promotes cytochrome c-dependent caspase activation by eliminating IAP inhibition. Cell 102: 33-42, 2000.

29. Verhagen AM, Ekert PG, Pakusch M, Silke J, Connolly LM, Reid GE, Moritz RL, Simpson RJ and Vaux DL: Identification of DIABLO, a mammalian protein that promotes apoptosis by binding to and antagonizing IAP proteins. Cell 102: 43-53, 2000

30. Li LY, Luo $X$ and Wang X: Endonuclease G is an apoptotic DNase when released from mitochondria. Nature 412: 95-99, 2001.

31. Baffy G, Miyashita T, Williamson JR and Reed JC: Apoptosis induced by withdrawal of interleukin-3 (IL-3) from an IL-3-dependent hematopoietic cell line is associated with repartitioning of intracellular calcium and is blocked by enforced Bcl-2 oncoprotein production. J Biol Chem 268: 6511-6519, 1993.
32. Hennet T, Bertoni G, Richter C and Peterhans E: Expression of BCL-2 protein enhances the survival of mouse fibrosarcoid cells in tumor necrosis factor-mediated cytotoxicity. Cancer Res 53: $1456-1460,1993$

33. Lam M, Dubyak G, Chen L, Nuñez G, Miesfeld RL and Distelhorst CW: Evidence that BCL-2 represses apoptosis by regulating endoplasmic reticulum-associated $\mathrm{Ca}^{2+}$ fluxes. Proc Natl Acad Sci USA 91: 6569-6573, 1994.

34. Zhang N, Wang X, Huo Q, Li X, Wang H, Schneider P, Hu G and Yang Q: The oncogene metadherin modulates the apoptotic pathway based on the tumor necrosis factor superfamily member TRAIL (tumor necrosis factor-related apoptosis-inducing ligand) in breast cancer. J Biol Chem 288: 9396-9407, 2013.

35. Crompton M: Bax, Bid and the permeabilization of the mitochondrial outer membrane in apoptosis. Curr Opin Cell Biol 12: 414-419, 2000.

36. Habibović S, Hrgović Z, Bukvić I and Hrgović I: Molecular mechanisms in apoptosis. Med Arh 54: 33-40, 2000 (In Croatian).

37. Antonsson B: Bax and other pro-apoptotic Bcl-2 family 'killer-proteins' and their victim the mitochondrion. Cell Tissue Res 306: 347-361, 2001

38. Himelstein BP, Lee EJ, Sato H, Seiki M and Muschel RJ: Tumor cell contact mediated transcriptional activation of the fibroblast matrix metalloproteinase-9 gene: Involvement of multiple transcription factors including Ets and an alternating purine-pyrimidine repeat. Clin Exp Metastasis 16: 169-177, 1998.

39. Hu Y and Ivashkiv LB: Costimulation of chemokine receptor signaling by matrix metalloproteinase- 9 mediates enhanced migration of IFN-alpha dendritic cells. J Immunol 176: 6022-6033, 2006.

40. Kallakury BV, Karikehalli S, Haholu A, Sheehan CE, Azumi N and Ross JS: Increased expression of matrix metalloproteinases 2 and 9 and tissue inhibitors of metalloproteinases 1 and 2 correlate with poor prognostic variables in renal cell carcinoma. Clin Cancer Res 7: 3113-3119, 2001.

41. Wang F, Zhang P, Yang L, Yu X, Ye X, Yang J, Qian C, Zhang X, Cui YH and Bian XW: Activation of toll-like receptor 2 promotes invasion by upregulating MMPs in glioma stem cells. Am J Transl Res 7: 607-615, 2015.

42. Zhang Z, Lv J, Lei X, Li S, Zhang Y, Meng L, Xue R and Li Z: Baicalein reduces the invasion of glioma cells via reducing the activity of p38 signaling pathway. PLoS One 9: e90318, 2014.

43. Fresno Vara JA, Casado E, de Castro J, Cejas P, Belda-Iniesta C and González-Barón M: PI3K/Akt signalling pathway and cancer. Cancer Treat Rev 30: 193-204, 2004.

44. Fang Z, Tang Y, Jiao W, Xing Z, Guo Z, Wang W, Shi B, Xu Z and Liu Z: Nitidine chloride inhibits renal cancer cell metastasis via suppressing AKT signaling pathway. Food Chem Toxicol 60: 246-251, 2013

45. Vier J, Groth M, Sochalska M and Kirschnek S: The anti-apoptotic Bcl-2 family protein A1/Bfl-1 regulates neutrophil survival and homeostasis and is controlled via PI3K and JAK/STAT signaling. Cell Death Dis 7: e2103, 2016.

46. Morgensztern D and McLeod HL: PI3K/Akt/mTOR pathway as a target for cancer therapy. Anticancer Drugs 16: 797-803, 2005

47. Carrera-González MP, Ramírez-Expósito MJ, Mayas MD and Martínez-Martos JM: Protective role of oleuropein and its metabolite hydroxytyrosol on cancer. Trends Food Sci Technol 31: 92-99, 2013. 